\title{
Anisotropic Coulomb effects on bremsstrahlung emission from bi-Maxwellian plasmas
}

Cite as: Journal of Applied Physics 91, 6188 (2002); https://doi.org/10.1063/1.1464205

Submitted: 19 October 2001 . Accepted: 04 February 2002 . Published Online: 17 April 2002

\section{Young-Dae Jung, and Chang-Geun Kim}
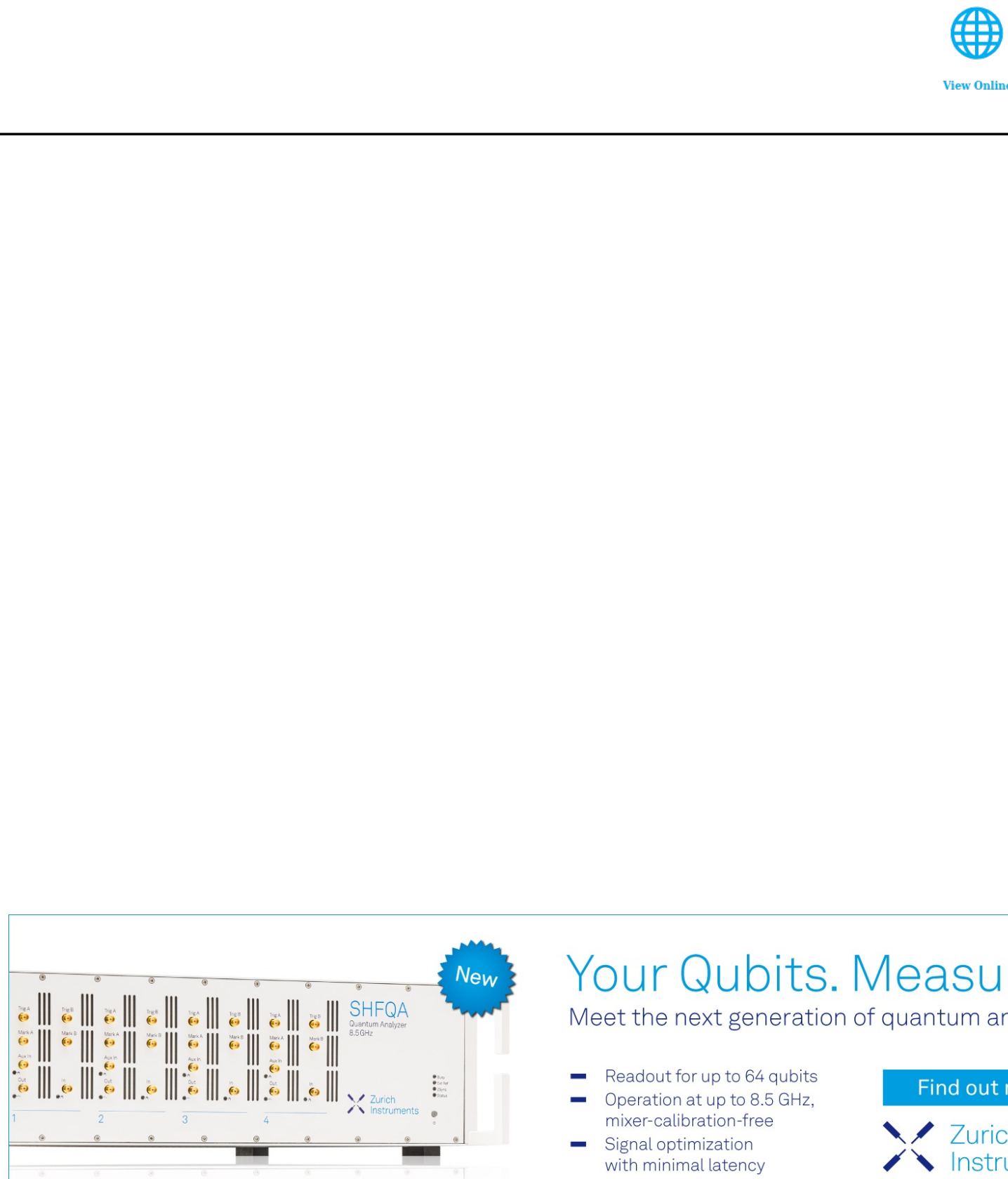

\section{Your Qubits. Measured.}

Meet the next generation of quantum analyzers

- Readout for up to 64 qubits

- Operation at up to $8.5 \mathrm{GHz}$.

Find out more

mixer-calibration-fre

- Signal optimization with minimal latency 


\title{
Anisotropic Coulomb effects on bremsstrahlung emission from bi-Maxwellian plasmas
}

\author{
Young-Dae Junga) \\ Department of Physics, Hanyang University, Ansan, Kyunggi-Do 425-791, South Korea \\ and Department of Physics, 0319, University of California, San Diego, 9500 Gilman Drive, \\ La Jolla, California 92093-0319 \\ Chang-Geun Kim \\ Department of Physics, Hanyang University, Ansan, Kyunggi-Do 425-791, South Korea
}

(Received 19 October 2001; accepted for publication 4 February 2002)

\begin{abstract}
Anisotropy of the electron-ion bremsstrahlung spectrum from bi-Maxwellian plasmas is investigated including the Coulomb correction effects. The Coulomb correction effects on the bremsstrahlung emission rate for $T_{\|}>T_{\perp}$ are found to be more significant than those for $T_{\|}<T_{\perp}$ where $T_{\|}$and $T_{\perp}$ are temperatures for the parallel and perpendicular motion to the magnetic field. An increase of the parallel or perpendicular temperature causes a decrease of the Coulomb effects on the bremsstrahlung emission power. (C) 2002 American Institute of Physics.
\end{abstract}

[DOI: $10.1063 / 1.1464205]$

The electron-ion Coulomb bremsstrahlung process $^{1-9}$ has been a subject of special attention in atomic and plasma physics $^{2,5,7,9}$ since the continuum $\mathrm{x}$-ray spectrum due to the bremsstrahlung process has long been used for plasma diagnostics. In the quantum-mechanical case, the nonrelativistic Bethe-Heitler formula ${ }^{1,4}$ for the electron-ion Coulomb bremsstrahlung cross section has been widely used for highenergy electron distributions. It is known that the BetheHeitler bremsstrahlung cross section approaches zero because of the inaccuracy of the Born approximation near the spectral cutoff region. However, the real electron-ion Coulomb bremsstrahlung cross section has to be finite at the cutoff spectrum. This inaccuracy near the cutoff spectral region can be removed by using the confluent hypergeometic functions for the initial and final states of the projectile electron. The Coulomb correction has been obtained by the Coulomb focusing ${ }^{6}$ factor without evaluating the full Hamiltonian transition matrix element using the confluent hypergeometric functions, especially, in hard spectral regions. This correction is quite important for evaluating the spectral bremsstrahlung emission rate. Many of the characteristic features of plasmas can be understood by knowing the velocity dependence of electron distributions. In many space and laboratory plasmas, the distribution function can be approximated by a bi-Maxwellian, which take into account the different velocities and temperatures of the two motions. ${ }^{10-12}$ Thus, in this article we investigate the first- and second-order Coulomb correction effects on the electron-ion Coulomb bremsstrahlung spectrum from a bi-Maxwellian distribution plasma. The electron-ion Coulomb bremsstrahlung cross section is given by the nonrelativistic BetheHeitler formula and the Sommerfeld Coulomb correction factor is also considered. The bremsstrahlung emission rate

\footnotetext{
a)Permanent address: Department of Physics, Hanyang University, Ansan, Kyunggi-Do 425-791, South Korea; electronic mail: yjung@bohr.hanyang.ac.kr and ydjung@ physics.ucsd.edu
}

from a bi-Maxwellian distribution plasma is also investigated for the radiation photon energy and tempatatures of the two motions.

For electron-ion Coulomb bremsstrahlung in the highenergy domain, the differential cross section ${ }^{6}$ can be obtained by the second-order perturbation theory with the plane wave Born approximations for the initial and final states of the projectile electron:

$$
\frac{d \sigma_{\mathrm{BH}}}{d \epsilon}=\frac{16}{3} \frac{Z^{2} \alpha r_{0}^{2} c^{2}}{\epsilon v^{2}} \ln \left(\frac{v+v^{\prime}}{v-v^{\prime}}\right),
$$

where $Z$ is the charge of the target ion, $\epsilon(\equiv \hbar \omega)$ is the bremsstrahlung photon energy, $\alpha\left(=e^{2} / \hbar c \approx 1 / 137\right)$ is the fine structure constant, $r_{0}\left(=e^{2} / m c^{2}\right)$ is the classical electron radius, $m$ is the mass of the electron, $v$ and $v^{\prime}$ $(=\sqrt{v-2 \hbar \omega / m})$ are the initial and final velocities of the projectile electron, respectively. This nonrelativistic Bethe-Heitler ${ }^{1}$ formula is known to be invalid for the final state of the projectile electron near the cutoff region $m v^{2} / 2$ $\approx \hbar \omega$. In order to correct the Bethe-Heitler cross section, we must consider the motion of the initial and final states of the projectile electron in the external field of the target ion using continuum wave function for the Coulomb potential. ${ }^{13}$ The Coulomb correction in the Hamiltonian transition matrix element can be well approximated by the ratio of the absolute square of the final Coulomb $s$-wave $(l=0)$ function $\left|\Psi_{f}(0)\right|^{2}\left[=\pi \beta e^{\pi \beta^{\prime}} / \sinh \left(\pi \beta^{\prime}\right), \beta^{\prime}=Z e^{2} / \hbar v^{\prime}\right]$ to the initial Coulomb $s$-wave wave function $\left|\Psi_{i}(0)\right|^{2}$ $\left[=2 \pi \beta e^{\pi \beta} / \sinh (\pi \beta), \beta=Z e^{2} / \hbar v\right]$ at the origin since $l=0$ survives for $r \rightarrow 0$ and the mutual Coulomb interaction between the electron and the target ion is quite effective for small separations due to the Coulomb focusing effect. This Coulomb focusing factor can be represented as

$$
F_{C}\left(\beta, \beta^{\prime}\right)=\frac{\beta^{\prime}}{\beta} \frac{1-e^{-2 \pi \beta}}{1-e^{-2 \pi \beta^{\prime}}} .
$$


In the Born limit, i.e., $\beta, \beta^{\prime} \ll 1, F_{C}\left(\beta, \beta^{\prime}\right) \rightarrow 1$. Thus, the bremsstrahlung emission rate for a given differential electron density distribution $d n_{e}(v)$ is given by

$$
P_{\epsilon}=\frac{d E_{\mathrm{rad}}}{d V d t d \epsilon}=\int d n_{e}(v) n_{i} v \epsilon \frac{d \sigma_{\mathrm{BH}}(v)}{d \epsilon} F_{c}\left(v, v^{\prime}\right),
$$

where $E_{\text {rad }}$ is the radiation energy and $n_{i}$ is the ion density.

Many laboratory and astrophysical plasmas have been found to have non-Maxwellian electron distribution. ${ }^{14,15}$ If a plasma has a difference in the velocities parallel and perpendicular to the magnetic field, the electron distribution can be approximated by a bi-Maxwellian which is the product of two Maxwellians. The bi-Maxwellian electron distribution ${ }^{10}$ $f\left(v_{\|}, v_{\perp}\right)$ is a typical anisotropic distribution function:

$$
\begin{aligned}
f\left(v_{\|}, v_{\perp}\right)= & \left(\frac{m}{2 \pi k_{B}}\right)^{3 / 2} \frac{n_{e}}{T_{\perp} \sqrt{T_{\|}}} \\
& \times \exp \left(-\frac{m v_{\|}^{2}}{2 \pi k_{B} T_{\|}}\right) \exp \left(-\frac{m v_{\perp}^{2}}{2 \pi k_{B} T_{\perp}}\right),
\end{aligned}
$$

where $k_{B}$ denotes the Boltzmann constant, $v_{\|}$and $v_{\perp}$ are the velocities parallel and perpendicular to the magnetic field, and $T_{\|}$and $T_{\perp}$ are temperatures for the parallel and perpendicular motion to the magnetic field. Then, the differential electron distribution function can be represented by

$$
d n_{e}\left(v_{\|}, v_{\perp}\right)=2 \pi d v_{\|} v_{\perp} d v_{\perp} f\left(v_{\|}, v_{\perp}\right) .
$$

This anisotropic distribution function $f\left(v_{\|}, v_{\perp}\right)$ is the one most commonly found in space plasmas. In the anisotropic Maxwellian, the contours in the momentum space are deformed into elliptic shapes and the deformation corresponds to $\Delta T\left(\equiv T_{\|}-T_{\perp}\right)$.

After some algebra using the relations $v_{\|}$ $=(2 \hbar \omega / m)^{1 / 2} \eta$ and $v_{\perp}=(2 \hbar \omega / m)^{1 / 2} \zeta$, the bremsstrahlung emission rate can also be written as

$$
\begin{aligned}
& P_{\epsilon}\left(k_{B} \bar{T}_{\perp}, k_{B} \bar{T}_{\|}\right) \\
& \quad=\frac{32}{3} \sqrt{\frac{2}{\pi}} \frac{Z^{2} \alpha r_{0}^{2} c^{2} n_{e} n_{i}}{k_{B} \bar{T}_{\perp} \sqrt{k_{B} \bar{T}_{\|}}} \bar{\epsilon} I\left(\bar{\epsilon}, k_{B} \bar{T}_{\perp}, k_{B} \bar{T}_{\|}\right),
\end{aligned}
$$

where $\bar{\epsilon} \equiv \hbar \omega / Z^{2} \mathrm{Ry}, \operatorname{Ry}\left(=m e^{4} / 2 \hbar^{2} \cong 13.6 \mathrm{eV}\right)$ is the Rydberg constant, $k_{B} \bar{T}_{\perp}\left(\equiv k_{B} T_{\perp} / Z^{2} \mathrm{Ry}\right)$ and $k_{B} \bar{T}_{\|}$ $\left(\equiv k_{B} T_{\|} / Z^{2} \mathrm{Ry}\right)$ are, respectively, scaled perpendicular and parallel temperatures. Here, the function $I\left(\bar{\epsilon}, k_{B} \bar{T}_{\perp}, k_{B} \bar{T}_{\|}\right)$is written as

$$
I\left(\bar{\epsilon}, k_{B} \bar{T}_{\perp}, k_{B} \bar{T}_{\|}\right)=\sum_{n=0}^{\infty} \frac{1}{|2 n-1|}\left(\frac{\pi}{\sqrt{\bar{\epsilon}}}\right)^{n} I_{n}\left(\bar{\epsilon}, k_{B} \bar{T}_{\perp}, k_{B} \bar{T}_{\|}\right),
$$

where $I_{0}\left(\bar{\epsilon}, k_{B} \bar{T}_{\perp}, k_{B} \bar{T}_{\|}\right)$is

$$
\begin{aligned}
I_{0}\left(\bar{\epsilon}, k_{B} \bar{T}_{\perp}, k_{B} \bar{T}_{\|}\right)= & \int_{\eta_{0}}^{\infty} d \eta \int_{\zeta_{0}}^{\infty} \zeta d \zeta \exp \left[-\left(\bar{\epsilon} / k_{B} \bar{T}_{\perp}\right) \zeta^{2}\right] \exp \left[-\left(\bar{\epsilon} / k_{B} \bar{T}_{\|}\right) \eta^{2}\right] \\
& \times \frac{1}{\left(\eta^{2}+\zeta^{2}\right)^{1 / 2}} \ln \left[\frac{\left(\eta^{2}+\zeta^{2}\right)^{1 / 2}+\left(\eta^{2}+\zeta^{2}-1\right)^{1 / 2}}{\left(\eta^{2}+\zeta^{2}\right)^{1 / 2}-\left(\eta^{2}+\zeta^{2}-1\right)^{1 / 2}}\right]
\end{aligned}
$$

with $\eta_{0}^{2}+\zeta_{0}^{2}=1$. Here, $I_{0}\left(\bar{\epsilon}, k_{B} \bar{T}_{\perp}, k_{B} \bar{T}_{\|}\right)$is the bremsstrahlung emission function without including any Coulomb corrections. Then, $I_{1}\left(\bar{\epsilon}, k_{B} \bar{T}_{\perp}, k_{B} \bar{T}_{\|}\right)$is the first-order Coulomb correction, i.e., the first-order Coulomb focusing, to $I_{0}\left(\bar{\epsilon}, k_{B} \bar{T}_{\perp}, k_{B} \bar{T}_{\|}\right)$:

$$
\begin{aligned}
I_{1}\left(\bar{\epsilon}, k_{B} \bar{T}_{\perp}, k_{B} \bar{T}_{\|}\right)= & \int_{\eta_{0}}^{\infty} d \eta \int_{\zeta_{0}}^{\infty} \zeta d \zeta \exp \left[-\left(\bar{\epsilon} / k_{B} \bar{T}_{\perp}\right) \zeta^{2}\right] \exp \left[-\left(\bar{\epsilon} / k_{B} \bar{T}_{\|}\right) \eta^{2}\right] \\
& \times \frac{1}{\left(\eta^{2}+\zeta^{2}\right)^{1 / 2}} \ln \left[\frac{\left(\eta^{2}+\zeta^{2}\right)^{1 / 2}+\left(\eta^{2}+\zeta^{2}-1\right)^{1 / 2}}{\left(\eta^{2}+\zeta^{2}\right)^{1 / 2}-\left(\eta^{2}+\zeta^{2}-1\right)^{1 / 2}}\right]\left[\frac{1}{\left(\eta^{2}+\zeta^{2}-1\right)^{1 / 2}}-\frac{1}{\left(\eta^{2}+\zeta^{2}\right)^{1 / 2}}\right],
\end{aligned}
$$

and $I_{2}\left(\bar{\epsilon}, k_{B} \bar{T}_{\perp}, k_{B} \bar{T}_{\|}\right)$is the second-order Coulomb correction, i.e., the second-order Coulomb focusing, to $I_{0}\left(\bar{\epsilon}, k_{B} \bar{T}_{\perp}, k_{B} \bar{T}_{\|}\right)$:

$$
\begin{aligned}
I_{2}\left(\bar{\epsilon}, k_{B} \bar{T}_{\perp}, k_{B} \bar{T}_{\|}\right)= & \int_{\eta_{0}}^{\infty} d \eta \int_{\zeta_{0}}^{\infty} \zeta d \zeta \exp \left[-\left(\bar{\epsilon} / k_{B} \bar{T}_{\perp}\right) \zeta^{2}\right] \exp \left[-\left(\bar{\epsilon} / k_{B} \bar{T}_{\|}\right) \eta^{2}\right] \\
& \times \frac{1}{\left(\eta^{2}+\zeta^{2}\right)^{1 / 2}} \ln \left[\frac{\left(\eta^{2}+\zeta^{2}\right)^{1 / 2}+\left(\eta^{2}+\zeta^{2}-1\right)^{1 / 2}}{\left(\eta^{2}+\zeta^{2}\right)^{1 / 2}-\left(\eta^{2}+\zeta^{2}-1\right)^{1 / 2}}\right]\left[\frac{2}{\eta^{2}+\zeta^{2}-1}+\frac{1}{\eta^{2}+\zeta^{2}}-\frac{3}{\left(\eta^{2}+\zeta^{2}-1\right)^{1 / 2}\left(\eta^{2}+\zeta^{2}\right)^{1 / 2}}\right] .
\end{aligned}
$$

Figures 1 and 2 show the first $\left(I_{1} / I_{0}\right)$ - and second $\left(I_{2} / I_{0}\right)$-order Coulomb corrections on the electron-ion bremsstrahlung emission rate as functions of the scaled radiation photon energy. As we can see in Figs. 1 and 2, the Coulomb corrections, i.e., the Coulomb focusing effects, on the bremsstrahlung spectrum decrease with increasing the perpendicular temperature for a given parallel temperature. Likewise, the Coulomb effects also decrease with increasing the parallel temperature for a fixed perpendicular temperature. It is also found that the Coulomb correction effects on 


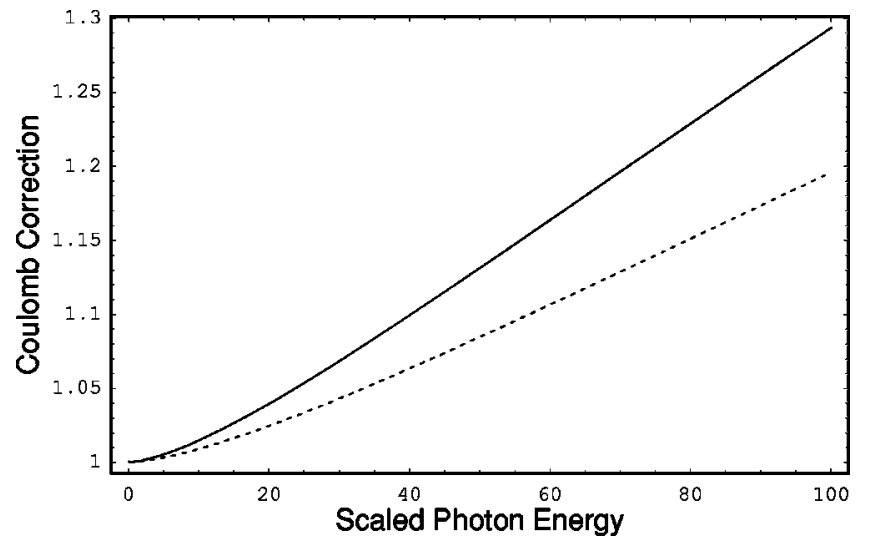

FIG. 1. First- and second-order Coulomb corrections on the bremsstrahlung emission rate $\left[1+(\pi / \sqrt{\bar{\epsilon}})\left(I_{1} / I_{0}\right)+\left(\pi^{2} / 3 \bar{\epsilon}\right)\left(I_{2} / I_{0}\right)\right]$ when $\eta_{0}=0$ and $\zeta_{0}$ $=1$. The solid line represents the bremsstrahlung emission rate for $k_{B} \bar{T}_{\|}$ $=100$ and $k_{B} \bar{T}_{\perp}=50$. The dotted line represents the bremsstrahlung emission rate for $k_{B} \bar{T}_{\|}=50$ and $k_{B} \bar{T}_{\perp}=100$.

the bremsstrahlung emission rate for $T_{\|}>T_{\perp}$ are more significant than those for $T_{\|}<T_{\perp}$, for example, when $\eta_{0}=0$ and $\zeta_{0}=1$, the Coulomb corrections are, respectively, 1.46 for $k_{B} \bar{T}_{\|}=50, k_{B} \bar{T}_{\perp}=25$, and $\bar{\epsilon}=50$ and 1.31 for $k_{B} \bar{T}_{\|}$ $=25, k_{B} \bar{T}_{\perp}=50$, and $\bar{\epsilon}=50$ and when $\eta_{0}=1$ and $\zeta_{0}=0$, the Coulomb effects are, respectively, 1.27 for $k_{B} \bar{T}_{\|}=50, k_{B} \bar{T}_{\perp}$ $=25$, and $\bar{\epsilon}=50$ and 1.24 for $k_{B} \bar{T}_{\|}=25, k_{B} \bar{T}_{\perp}=50$. This anisotropy is due to the difference in number of degrees of freedom for the parallel and the perpendicular motion. There is one degree of freedom parallel to the magnetic field while there are two perpendiculars to the field. The Coulomb cor-

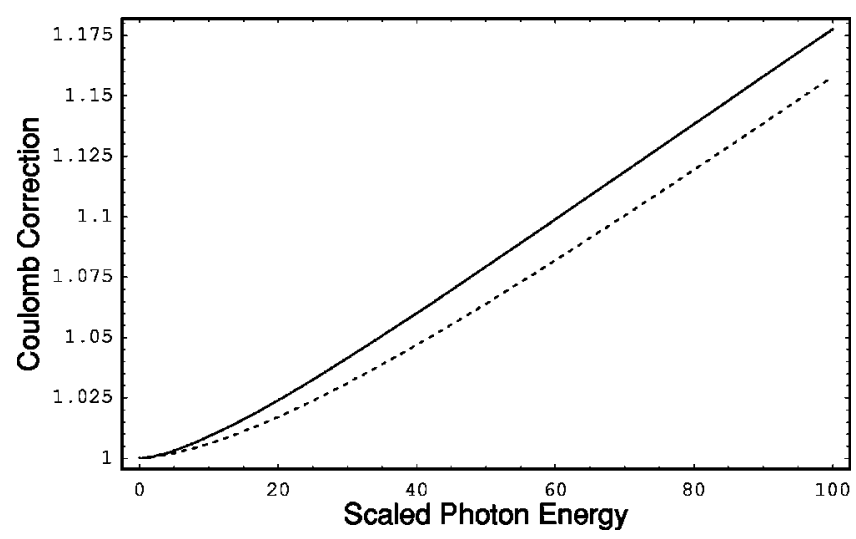

FIG. 2. First- and second-order Coulomb corrections on the bremsstrahlung emission rate $\left[1+(\pi / \sqrt{\bar{\epsilon}})\left(I_{1} / I_{0}\right)+\left(\pi^{2} / 3 \bar{\epsilon}\right)\left(I_{2} / I_{0}\right)\right]$ when $\eta_{0}=1$ and $\zeta_{0}$ $=0$. The solid line represents the bremsstrahlung emission rate for $k_{B} \bar{T}_{\|}$ $=100$ and $k_{B} \bar{T}_{\perp}=50$. The dotted line represents the bremsstrahlung emission rate for $k_{B} \bar{T}_{\|}=50$ and $k_{B} \bar{T}_{\perp}=100$. rections can also be understood through the behavior of the continuum wave function. The Eikonal-Coulomb (EC) wave function ${ }^{16} \Psi_{\mathrm{EC}}(\mathbf{r})$ using the Green's function of the Helmholtz equation ${ }^{17}$ is given by

$$
\Psi_{\mathrm{EC}}(\mathbf{r})=(2 \pi)^{-3 / 2} \exp [i \mathbf{k} \cdot \mathbf{r}-i \chi(\mathbf{k}, \mathbf{r})],
$$

where $\chi(\mathbf{k}, \mathbf{r})$ is the eikonal phase:

$$
\chi(\mathbf{k}, \mathbf{r})=\frac{m}{\hbar^{2} k} \int_{-\infty}^{r_{\|}} d r_{\|} V\left(r_{\|}, r_{\perp}\right),
$$

where $\mathbf{k}$ is the wave vector, and the perpendicular and parallel positions are represented by $r_{\|} \cong v_{\|} t$ and $r_{\perp} \cong v_{\perp} t$, respectively. Hence, an increase of the parallel or perpendicular temperature causes a decrease of the Coulomb effects on the bremsstrahlung emission power because of the eikonal phase $\chi(\mathbf{k}, \mathbf{r})$. It is obvious to found that the Coulomb correction effects are important for high photon energies since the energy of the final electron is soft for hard spectral regions. It has been found that the bremsstrahlung radiation cross section is increased with an increase of the cyclotron energy, i.e., the strength of the magnetic field. ${ }^{18}$ Thus, it is expected that the bremsstrahlung emission power would be enhanced with increasing the strength of the magnetic field.

One of the authors (Y.-D. Jung) gratefully acknowledges Professor R. J. Gould for warm hospitality while visiting the University of California, San Diego. He also thanks Dr. M. Rosenberg for useful discussions on bremsstrahlung processes. This work was supported by the Korean Ministry of Education through Brain Korea (BK21) Project and by the Korea Basic Science Institute through HANBIT User Development Program (FY2002).

${ }^{1}$ H. A. Bethe and E. E. Salpeter, Quantum Mechanics of One- and TwoElectron Atoms (Springer, Berlin, 1957).

${ }^{2}$ G. Bekefi, Radiation Processes in Plasmas (Wiley, New York, 1966).

${ }^{3}$ R. J. Gould, Astrophys. J. 238, 1026 (1980).

${ }^{4}$ V. B. Berestetskii, E. M. Lifshitz, and L. P. Pitaevskii, Quantum Electrodynamics, 2nd ed. (Pergamon, Oxford, 1982).

${ }^{5}$ I. H. Hutchinson, Principles of Plasma Diagnostics (Cambridge University Press, Cambridge, UK, 1987).

${ }^{6}$ R. J. Gould, Astrophys. J. 362, 248 (1990).

${ }^{7}$ V. N. Tsytovich, Lectures on Non-Linear Plasma Kinetics (Springer, Berlin, 1995).

${ }^{8}$ F. Melia, Electrodynamics (University of Chicago Press, Chicago, 2001).

${ }^{9}$ Y. D. Jung, Phys. Plasmas 1, 785 (1994).

${ }^{10}$ M.-B. Kallenrode, Space Physics, 2nd ed. (Springer, Berlin, 2001).

${ }^{11}$ M. A. M. ElSabbagh, M. D. Bowden, K. Uchino, and K. Muraoka, Appl. Phys. Lett. 78, 3187 (2001).

${ }^{12}$ E. Stamate and K. Ohe, J. Appl. Phys. 89, 2058 (2001).

${ }^{13}$ J. Schwinger, Quantum Mechanics (Springer, Berlin, 2001).

${ }^{14}$ J. Zheng, C. X. Yu, and Z. J. Zheng, Phys. Plasmas 4, 2736 (1997).

${ }^{15}$ A. Bandyopadhyay and K. P. Das, J. Plasma Phys. 65, 131 (2001).

${ }^{16}$ Y.-D. Jung, Phys. Lett. A 284, 275 (2001).

${ }^{17}$ J. Schwinger, L. L. DeRaad, Jr., K. A. Milton, and W.-Y. Tsai, Classical Electrodynamics (Perseus Books, Reading, MA 1998).

${ }^{18}$ Y.-D. Jung, Phys. Plasmas 7, 3181 (2000). 